\title{
Microcirculation Perfusion Monitor on the Back of the Health Volunteers
}

\author{
Yanqi Li, ${ }^{1}$ Xiaomei Li, ${ }^{2}$ Dan Zhou, ${ }^{1}$ Kang Wang, ${ }^{3}$ Yangyang Liu, ${ }^{1}$ Yi Guo, ${ }^{1}$ Shuang Qiu, ${ }^{4}$ \\ Tianchen Zhai, ${ }^{4}$ Shuang Liu, ${ }^{4}$ Jingjing Liu, ${ }^{4}$ and Dong Ming ${ }^{4}$ \\ ${ }^{1}$ Experimental Acupuncture Research Center of Tianjin University of Traditional Chinese Medicine, Tianjin 300193, China \\ ${ }^{2}$ Shenzhen Renren Health Management Co., LTD, Shenzhen 518000, China \\ ${ }^{3}$ The 2nd Clinical Medical College of Beijing University of Chinese Medicine, Beijing 100078, China \\ ${ }^{4}$ Neural Engineering \& Rehabilitation Lab of Tianjin University, Tianjin 300072, China \\ Correspondence should be addressed to Yi Guo; guoyi_168@163.com
}

Received 27 September 2013; Revised 1 November 2013; Accepted 15 November 2013

Academic Editor: Calvin Yu-Chian Chen

Copyright (c) 2013 Yanqi Li et al. This is an open access article distributed under the Creative Commons Attribution License, which permits unrestricted use, distribution, and reproduction in any medium, provided the original work is properly cited.

Objective. To observe the dermal microcirculation blood perfusion characterization of meridians channels (acupoints). Methods. 20 healthy human subjects were monitored using Pericam Perfusion Speckle Imager (PSI) for the changes in dermal microcirculation blood perfusion on governor meridian and other respective dermal regions as a control. Result. The microcirculation blood perfusion on Governor Meridian is higher than its control area. Conclusion. The dermal microcirculation blood perfusion on certain parts of Governor Meridian of healthy human subjects showed specifics.

\section{Introduction}

The meridian doctrine is one of the core theories of traditional Chinese medicine. After a half century's exploring and studying, prominent achievements have been gained in the field of the meridian doctrine and the specificity of meridians has been proved from various aspects. Biological characteristics of the meridians (acupoints) were monitored physiologically and pathologically by Contemporary scholars with many methods, including LDF and infrared spectrum detection [1]. For example, under the physical condition, ATP $[2,3]$, the oxygen partial pressure [4-9], transcutaneous $\mathrm{CO}_{2}$ emission (TCE) [10-17], temperature, and the microcirculation perfusion [18-31] at the acupoints were higher than that at the nonacupoints. Meanwhile, the corresponding indexes changed after the intervention measures like acupuncture stimulation, moxibustion, and pressure. Xu et al. [24] had found out that the dermal areas and deep tissues along the governor meridian had a higher microcirculation blood perfusion than nonmeridian controlled dermal regions on the healthy adults. In addition, the usage of electroacupuncture would result in a higher microcirculation blood perfusion.
However, the current researches now were mainly focused on the distinction between the acupoints and nonacupoints instead of the meridians and nonmeridians.

At present, most of the studies on the microcirculation were proceeded with the LDF. However, the LDF had its own limitations that could not achieve a large area and real time simultaneous monitoring at the same time. This study was carried on with the PeriCam PSI System (PSI). It is a blood perfusion imager based on the Laser Speckle Contrast Analysis (LASCA) technology. It is a method that visualizes tissue blood perfusion in real-time and combine dynamic response and spatial resolution in one instrument, providing both real-time graphs and video recordings of the area of interest. LASCA provides new means to study the microcirculation in ways that were not possible in the past.

Nonlinear analysis has been applied more and more in the microcirculation analysis and provided strong supports for the microcirculation researches. Several nonlinear analysis strategies, such as fractal analysis [32] and complexity analysis [33] on the microcirculation signals, provided statistical evidence for the further understanding of the microcirculation either under the physiological or pathological 

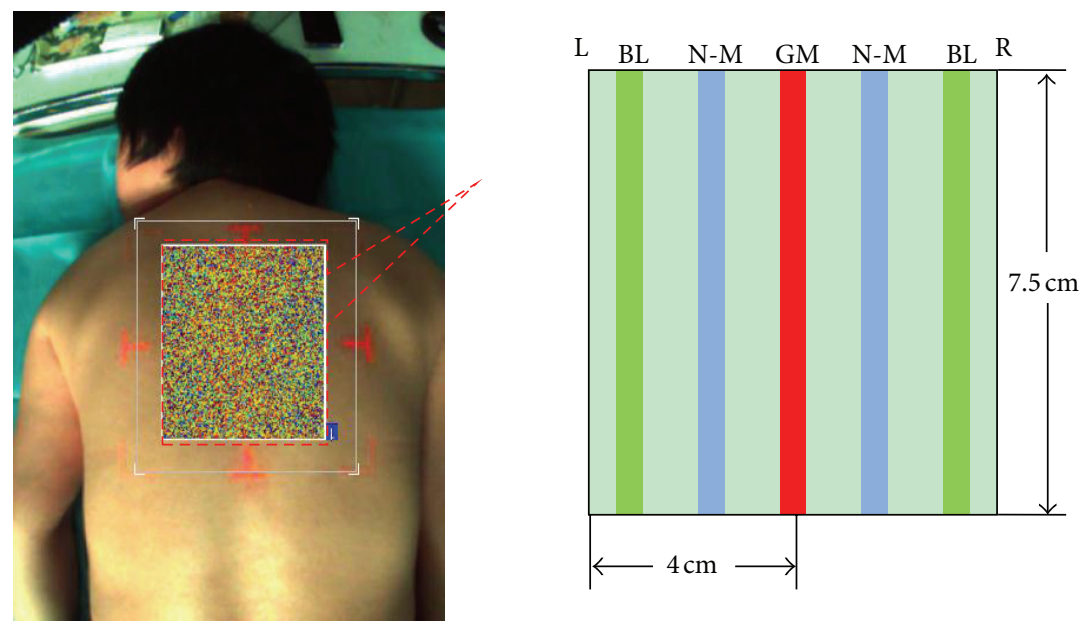

FIGURE 1: Diagram for the data acquisition.

conditions. The energy amount by Fourier transformation, support vector machine (SVM), and the fuzzy c-means algorithm are applied in this study. All of the three methods can describe and provide ample biological information in the microcirculation characters and provide evidences from different aspects.

The purpose of this study is to monitor the blood of the microcirculation in the back with the PeriCam PSI System (PSI) in a large area and real time, analyze data by nonlinear analysis, and compare the blood features between the governor meridian and nonmeridians so as to confirm that whether there is a difference in the dermal blood perfusion along the governor meridian.

\section{Methods}

2.1. Ethics Statement. This study was authorized by the Institutional Review Board of Acupuncture and Moxibustion institution, Tianjin University of Traditional Chinese Medicine (TCM). Each participant approved and signed the informed consent form before the study.

2.2. Subjects. Our study was conducted at the experimental acupuncture research center of Tianjin University of TCM between June and September, 2012. 20 healthy participants (10 males and 10 females) aged from 24 to 28 were recruited from Tianjin University of TCM. Smokers and alcoholics, people under recent emotional stress or have taken vasoactive drugs that may affect microcirculation, and people with skin lesions such as scars and acne on the experimental region were excluded. Participants were all informed and agreed to our study procedures.

\subsection{Methodology}

2.3.1. Experiment Conditions. In our study, room temperature was controlled around $26 \pm 1^{\circ} \mathrm{C}$, and the relative humidity was maintained at $50 \%-60 \%$. No direct sunlight or obvious indoor air convection was allowed during experiment.
The PSI parameter was set as follows: sample rate of 1 frame per second and detecting distance of $20 \pm 1 \mathrm{~cm}$ with the whole monitoring area of $14 \times 14 \mathrm{~cm}^{2}$. The monitoring area was also called the Region of Interest (ROI); in this study, we chose T5 as the center of the ROI. Perfusion data was recorded in real time, and the perfusion unit (PU) of the ROI was calculated and finally analyzed by the PSI System.

2.3.2. Location. We chose governor meridian (GM) and the two medial branches of bladder meridian (BM) as the study group; meridians were located according to the international standard. Besides, two nonmeridians (N-M), the middle line between the governor meridian, and the medial branch of bladder meridian were cited as the control group (Figure 1).

2.3.3. Experiment Flow. ROI was marked and exposed to air before sampling so as to adapt to the environment; participants were in prone position and told to be relaxed. 20 min later, the blood perfusion data of the marked spot was recorded for $10 \mathrm{~min}$.

2.3.4. Statistics. All data was transformed, calculated, and compared using Fourier transformation, support vector machine, and fuzzy c-means algorithm.

\section{Experiment Results}

3.1. Experiment Subjects. 20 healthy participants were included from June to September, 2012, and all of them completed the study.

3.2. Data Process. An area of $7.5 \times 8 \mathrm{~cm}^{2}$ of the ROI was chosen for analysis, which was basically from T2 to T9. GM, $\mathrm{BL}$, and N-M (the midline of the governor meridian and bladder meridian) were marked in this filed. For further data analysis, each rectangle was separated into 15 points with $0.5 \mathrm{~cm}$ diameter, respectively (Figure 2 ). 


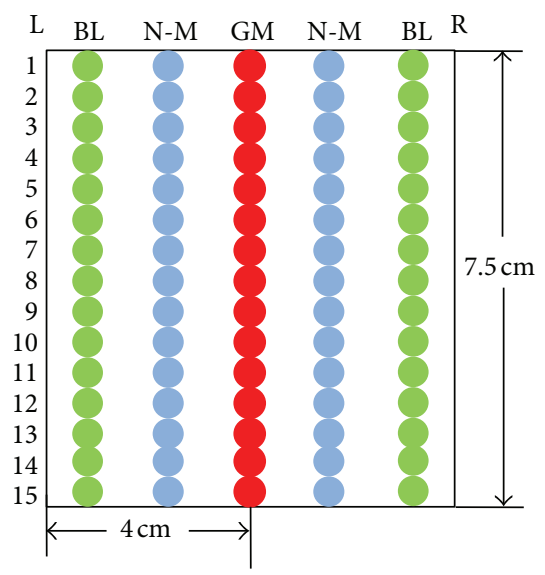

FiguRE 2: Diagram for the data acquisition.

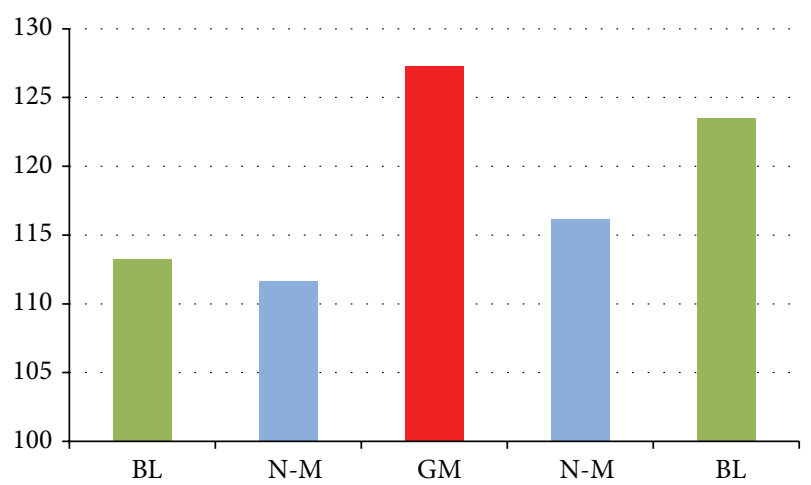

FIgURE 3: Microcirculation perfusion monitor on the back of the health volunteers. $P_{\mathrm{GM} \text { : left BL }}=0.061 ; P_{\mathrm{GM}}$ : right $\mathrm{BL}=0.679$; $P_{\mathrm{GM}}$ left N-M $=0.014 ; P_{\mathrm{GM}: \text { right N-M }}=0.016$.

3.2.1. Energy Amount by Fourier Transformation. Based on the theory of Fourier transformation, the acquired perfusion data was transformed into energy signals. The amount of energy of the five groups was calculated, respectively, and then compared (Figure 3).

The result of the energy amount showed that the governor meridian had the highest amount of energy, which followed by the medial branches of the bladder meridian and the nonmeridians. Moreover, the meridian and nonmeridian on the right side had higher energy than the left side. When compared statistically, there was significant statistical difference between the governor meridian and the two nonmeridians, while no statistical difference was observed between the governor meridian and the two bladder meridians or between the two bladder meridians and the nonmeridians. Therefore, the microcirculation blood perfusion on the governor meridian was obviously more activated than the other four lines.

3.2.2. Support Vector Machine (SVM). Support vector machine (SVM) is a kind of pattern recognition method based on statistical theories; it helps building a higher-dimensional space, the maximum separation hyperplane, to present all the vector data. In our study, we used this method to map the

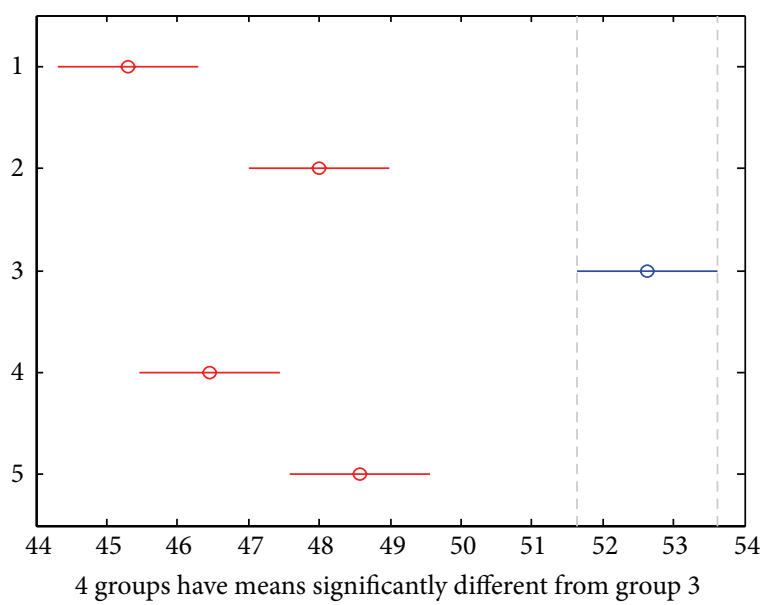

FIGURE 4: Microcirculation perfusion monitor on the back of the health volunteers; 1 denotes the left medial branch of the BL meridian; 2 denotes the left control line; 3 denotes the right control line; and 4 denotes the right medial branch of the BL meridian.

perfusion data of the five lines (governor meridian, bilateral medial branches of the bladder meridian, and the nonmeridians) into the maximum separation hyperplane. In this higherdimensional space (Figure 4), overlapped vectors stand for no statistical difference, such as the two nonmeridians; while disoverlapped vectors imply for the existence of statistical differences, such as the governor meridian and nonmeridian. Therefore, we found out that the governor meridian had higher perfusion than either the medial branch of the bladder meridian or the nonmeridian practically and statistically.

3.2.3. Fuzzy c-Means Algorithm (FCM). Fuzzy c-means algorithm (FCM) was an algorithm which divides the limit numbered data into different clusters using membership function and the iterative algorithm on the premise of a defined cluster number. There were 3 clustering centers in each matrix, therefore, together $N=45(15 \times 3)$ samplings were analyzed in 20 dimensions. Perfusion data was finally output into $45 \times 20$ matrices, and each matrix represented the average blood perfusion of the sampling area. We mapped all the matrixes into a 20-dimensional coordinate as shown in Figures 5 and 6.

Governor meridian and the two medial branches of the bladder meridian were cited as the 3 clustering centers and samplings on governor meridian; the left and right medial branch of the bladder meridian were marked into green, blue, and red, respectively. By using FCM, a 20-dimensional chart of the 3 clustering centers was generated (as shown in Figure 5), and separated samplings in this chart stood for statistically difference of the corresponding meridians. It is clear that the PU of the green samplings (governor meridian) is significantly different from the other two colors (two bladder meridians), which means that the PU of governor meridian is statistically different from the two bladder meridians, while there is no difference between the two bladder meridians.

Use the same method to compare governor meridian with bilateral nonmeridians; similarly, mark governor meridian 


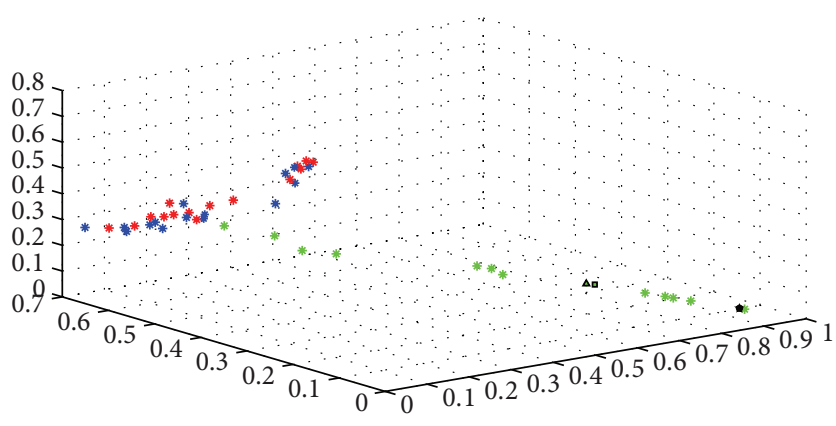

Figure 5: Microcirculation perfusion monitor on the back of the health volunteers. Green ones denote the governor meridian; blue ones denote the left medial branch of the BL meridian; and red ones denote the right medial branch of the BL meridian.

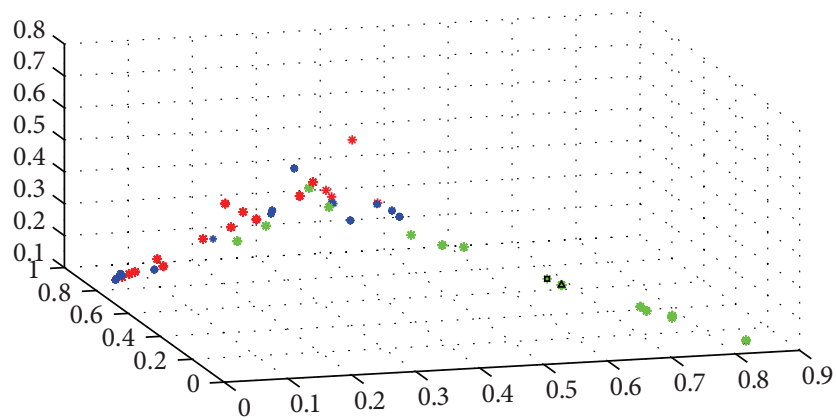

FIGURE 6: Microcirculation perfusion monitor on the back of the health volunteers. Green ones denote the governor meridian; blue ones denote the left control line; abd red ones denote the right control line.

and the left and right nonmeridian into green, blue, and red (as shown in Figure 6). The PU of the governor meridian was significantly statistically different from the two nonmeridians, while no difference was observed between the two nonmeridians.

\section{Discussion}

4.1. Results. Our study showed that governor meridian had a better microcirculation blood perfusion in healthy adults and its perfusion was higher than not only the two bilateral medial bladder meridians but also the nonmeridians, which reflected the characteristics of the microcirculation of the governor meridian directly. And the results were consistent with the previous researches [23].

The microcirculation of meridians is affected by numerous factors; the permeability of capillaries as well as vasoactive agent is of great importance. During the prestage of our study, we [34] found that, by injecting EB coloring agent into the veins surrounding the ears of healthy rabbits, the EB exudation along the governor meridian on the back was higher than nonmeridian lines on the bilateral of governor meridian; it indicated that the blood vessels along the governor meridian have exceptionally high permeability. Moreover, other researchers have shown that chemicals which affect the activation of blood vessels such as CGRP, Substance P, and NO might have their own respective dispersion characteristics. For example, a study by $\mathrm{Ma}$ et al. [35] on the correlation between temperature changes in tissues along meridians and CGRP concentration has found that there was higher CGRP concentration in tissues along meridians with elevated temperature than in area without elevated temperatures. Cao et al. [36] have shown that electrical stimulation of "Zusanli" acupoint on rats resulted in elevated SP concentrations in the dermal layer along the stomach meridian as well as in the colon, indicating that SP is involved in both the meridian activity from acupoint stimulation and the response of the respective organ. Ma [37] has found that NO concentration is significantly higher in tissues of acupoints; histochemistry test has shown that there is elevated NO expression in nerve fibers, axons, neurons, and hair follicles of acupoints.

4.2. Statistical Method. In recent years, the use of bioinformatic and nonlinear dynamic analysis methods has been increasingly widespread in medical field [38-40]. For example, Li et al. [41] used bioinformatic analysis methods to illustrate the tonifying and reduction techniques from a microcirculation point of view, which effectively distinguished the difference between the lifting-thrusting reinforcing method and lifting-thrusting reducing method of acupuncture. Wang et al. [42] used system identification algorithm to obtain biological characteristics of both left and right "he gu" acupoint, demonstrating the single side nature of acupoints. In our study, we applied the nonlinear dynamic method to analyze the perfusion data. This method was also successfully used in our other researches to analyze the effect of acupuncture on nerve impulses [43] and have found that the nerve tracts discharging sequence responding to different acupuncture techniques might have respective characteristic and could be differentiated by "time span," "frequency," and so forth, which was an elementary step to the establishment of a scientific description of the various acupuncture techniques.

In this study, multiple nonlinear analyzing methods were applied in order to distinguish the features of the governor meridian and nonmeridian from different views and verify the specificity of the microcirculation in the physiological status, which is suitable for this study. The application of nonlinear analysis could supplement the limitations of the general medical statistical analysis and is conductive to mining more biological information, which may explain the biological meanings reflected by the experimental data.

At present, we have observed part of the characteristics of the dermal microcirculation blood perfusion of the governor meridian. The whole features of the governor meridian have not been shown. In the further research, we plan to accomplish the whole features of the governor meridian as well as other twelve regular meridians and draw the specific dermal microcirculation blood perfusion chromatogram of the fourteen meridians in order to provide evidences to the microcirculation blood changes after the interventions or under the pathological conditions. 


\section{Conclusion}

Governor meridian has the feature of a high microcirculation blood perfusion in healthy people.

\section{Conflict of Interests}

All of the authors declare that they have no conflict of interest.

\section{Acknowledgments}

This work is supported by the Grant (no. 81302916) from the National Natural Science Foundation of China. Sincerely, thanks goes to Zhang Yue for the text proofreading. Thanks goes to the team led by Professor Dong Ming from Neural Engineering \& Rehabilitation Lab of Tianjin University. Shuang Qiu, Tianchen Zhai, Shuang Liu, and Liujing from this team helped with the data analysis. Thank goes to all of the participants. Yanqi Li and Xiaomei Li planned the study, recruited subjects, and performed the experiments. Yanqi Li and Kang Wang finished the paper. Yangyang Liu and Dan Zhou provided advices on the experiments and the paper. Yi Guo was the authority for the study planning as well as giving advice on the experiment.

\section{References}

[1] H. Hsiu, S. M. Huang, P. T. Chao et al., "Study on the microcirculatory blood velocity of acupoint monitored by laser Dopplesignal," in Proceedings of the IEEE Engineering in Medicine and Biology Society Conference, 2007.

[2] L.-H. Tan, C.-H. Ma, D. Zhang, S.-Y. Wang, and H.-M. Ma, "Changes of cutaneous temperature and the activity of $\mathrm{Ca}^{2+}$ and $\mathrm{Mg}^{2+}$-ATPase in the tissues along the running course of meridians in rabbit," Acupuncture Research, vol. 32, no. 5, pp. 330-333, 2007.

[3] Q. H. Han, G. H. Ding, and X. Y. Shen, "Infrared radicalization spectrum of human surface and the relationship with ATP energy metabolize," Shanghai Journal of Biomedical Engineering, vol. 26, no. 4, pp. 198-200, 2005.

[4] H. Wang, H. P. Zhang, and Y. X. Liu, "Test and analysis on deep meridian oxygen partial pressure," Hubei College of Traditional Chinese Medicine, vol. 16, no. 2, pp. 30-31, 1994.

[5] M. Chen, Z. X. Wu, X. L. Hu, and J. S. Xu, "Effect of acupuncture on partial oxygen pressure and temperature of deep tissues along large intestine channel in 30 normal volunteer subjects," Chinese Journal of Basic Medicine in Traditional Chinese Medicine, no. 5, pp. 553-555, 2011.

[6] M. Chen, X.-L. Hu, and Z.-X. Wu, "Effect of acupuncture on partial oxygen pressure of deep tissue along the Governor Vessel in 31 normal volunteer subjects," Acupuncture Research, vol. 33, no. 6, pp. 402-405, 2008.

[7] Y. J. Xie, X. L. Hu, and B. H. Wu, "Observation on the tissue $\mathrm{pO}_{2}$ after acupuncture," Chinese Journal of Traditional Medical Science and Technology, vol. 7, no. 6, pp. 353-354, 2000.

[8] H. Wang, H. P. Zhang, Y. X. Liu, Y. M. Chen, and Z. H. Liang, "Observation on the $\mathrm{po}_{2}$ of the acupoints off the acupoint on the meridian and off the meridian," Shanghai Journal of Acupuncture and Moxibustion, vol. 16, no. 1, pp. 3-5, 1997.
[9] X. Y. Xu, X. L. Hu, and B. H. Wu, "Influence of mechanical pressing on partial pressure of oxygen in three points along the large intestine meridian during acupuncture," Acupuncture Research, vol. 25, no. 4, pp. 276-279, 2000.

[10] A. Eory, "In vivo skin respiration $\left(\mathrm{CO}_{2}\right)$ measurements in the acupuncture loci," Acupuncture and Electro-Therapeutics Research, vol. 9, no. 4, pp. 217-223, 1984.

[11] "The phenomena expressed by skin respiration $\left(\mathrm{CO}_{2}\right.$ emission) along the lung channel," Compilation of the Abstracts of Acupuncture Moxibustion Paper, Beijing, China: WFAS, 1987.

[12] W. B. Zhang, X. H. Jing, R. M. Xu, Z. X. Zhu, C. H. Li, and H. $\mathrm{Li}$, "Characters on the Li meridian and the ST meridian on the of tagmata derma," Chinese Journal of Integrated Traditional and Western Medicine, vol. 15, no. 10, pp. 625-627, 1995.

[13] W. B. Zhang, H. Li, and R. M. Xu, "Observation of the TCE for the acupuncture on the meridian derma," Chinese Acupuncture \& Moxibustion, vol. 16, no. 1, pp. 39-42, 1996.

[14] W. B. Zhang and H. Li, "Research on the TCE mechanism on the dermal," Beijing Biomedical Engineering, vol. 15, no. 4, pp. 221-226, 1996.

[15] W.-B. Zhang, L.-L. Wang, T. Huang et al., "Laser Doppler perfusion imaging for assessment of skin blood perfusion after acupuncture," Medical Acupuncture, vol. 20, no. 2, pp. 109-118, 2008.

[16] R.-H. Wang, T. Huang, Y.-Y. Tian et al., "[Influence of mechanical pressure on the change of acupuncture-induced transcutaneous $\mathrm{CO}_{2}$ emission]," Chinese Acupuncture \& Moxibustion, vol. 29, no. 7, pp. 565-568, 2009.

[17] T. Huang, R. H. Wang, W. B. Zhang et al., "Relationship between needle sensation and periphery transcutaneous $\mathrm{CO}_{2}$ emission," Chinese Journal of Basic Medicine in Traditional Chinese Medicine, vol. 15, no. 8, pp. 615-618, 2009.

[18] X.-L. Hu, J.-S. Xu, P.-Q. Wang, X.-Y. Xu, L.-Y. Gao, and B.-H. $\mathrm{Wu}$, "Preliminary exploration on the mechanism underlying the formation of infrared radiant track along meridian courses over human body surface," Journal of Infrared and Millimeter Waves, vol. 22, no. 3, pp. 175-243, 2003.

[19] X.-L. Hu, P.-Q. Wang, J.-S. Xu, B.-H. Wu, and X.-Y. Xu, "Main characteristics of infrared radiant rack along meridian courses over human body surface and the condition of its appearance," Journal of Infrared and Millimeter Waves, vol. 20, no. 5, pp. 325328, 2001.

[20] X.-L. Hu, J.-S. Xu, L. Ye, J. Yang, P.-Q. Wang, and B.-H. Wu, "Elicitation of infrared radiant track along meridian courses over human body surface by local heating," Journal of Infrared and Millimeter Waves, vol. 21, no. 1, pp. 6-83, 2002.

[21] J.-S. Xu, X.-L. Hu, P.-Q. Wang, and B.-H. Wu, "Influence of electroacupuncture on infrared radiant track along meridian courses over human body surface," Chinese Journal of Clinical Rehabilitation, vol. 9, no. 29, pp. 251-253, 2005.

[22] J. S. Xu, X. Sh. Zheng, X. H. Pan, X. L. Hu, and Z. Y. Sa, "Influence of electro acupuncture on infrared radiant track along meridian courses over human body surface and microcirculation blood perfusion," Journal of Fujian University of Traditional Chinese Medicine, vol. 20, no. 1, pp. 13-73, 2010.

[23] J.-S. Xu, X.-H. Pan, X.-L. Hu, and B.-H. Wu, "Comparison between governor meridian and its bilateral control points in microcirculatory blood perfusion in 53 volunteer subjects," Acupuncture Research, vol. 33, no. 5, pp. 321-325, 2008.

[24] J. S. Xu, X. L. Hu, X. H. Pan, and S. Z. Sa, "Influence of electro acupuncture on infrared radiant track along meridian 
courses over human body surface and microcirculation blood perfusion," in Proceedings of the 12th National Infrared Heating and Infrared Medical Development Seminar Papers and Abstract Set, Nanjing, Infrared Electric Parts Professional Committee of Chinese Optical Society, pp. 22-25, 2009.

[25] D. Zhang, "Observation on the effects of moxibustion by laser Doppler perfusion imaging," Shanghai Journal of Acupuncture and Moxibustion, vol. 23, no. 5, pp. 37-40, 2004.

[26] D. Zhang, H. M. Ma, and S. H. .Y. Wang, "Infrared thermo gram observation on the blood circulation for the dermal temperature," Chinese Journal of Medical Imaging, vol. 9, no. 2, pp. 140-142, 2001.

[27] X. J. Wang, Ch.Ch. Zeng, H. P. Liu, S. H. Liu, and L. G. Liu, "The effects of laser acupuncture on temperature and blood flow perfusion rate of the point tissue," Acta Laser Biology Sinica, vol. 14, no. 4, pp. 260-264, 2005.

[28] H. Zhao, W. B. Zhang, and F. Y. Zhuang, "Effect of acupuncture at Zuanli on subcutaneous microcirculatory flow at point along meridian," Chinese Journal of Microcirculation, vol. 8, no. 1, pp. 41-45, 1998.

[29] H. Hsiu, W.-C. Hsu, B.-H. Chen, and C.-L. Hsu, "Differences in the microcirculatory effects of local skin surface contact pressure stimulation between acupoints and nonacupoints: possible relevance to acupressure," Physiological measurement, vol. 31, no. 6, pp. 829-841, 2010.

[30] G. Litscher, L. Wang, G. Schwarz, and D. Schikora, "Increases of intracranial pressure and changes of blood flow velocity due to acupressure, needle and laserneedle acupuncture?" Forschende Komplementarmedizin und Klassische Naturheilkunde, vol. 12, no. 4, pp. 190-195, 2005.

[31] K. Kubo, H. Yajima, M. Takayama, T. Ikebukuro, H. Mizoguchi, and N. Takakura, "Changes in blood circulation of the contralateral achilles tendon during and after acupuncture and heating," International Journal of Sports Medicine, vol. 32, no. 10, pp. 807813, 2011.

[32] F. Esen, G. S. Aydin, and H. Esen, "Detrended fluctuation analysis of laser Doppler flowmetry time series," Microvascular Research, vol. 78, no. 3, pp. 314-318, 2009.

[33] H. Hsiu, W. CH. Hsu, C. H. .L. Hsu, J. G. Bau, C. H. .T. Chen, and Y. S. Liu, "Complexity analysis of the microcirculatory-bloodflow response following acupuncture stimulation," Microvascular Research, no. 89, pp. 34-39, 2013.

[34] D. Zhou, Y. Guo, Y.-M. Guo, S. Zhang, and P. Pan, "Distribution and permeability of capillaries at the skin of the conception vessel and the governor vessel in healthy rabbits," Journal of Traditional Chinese Medicine, vol. 31, no. 4, pp. 356-359, 2011.

[35] C. H. H. Ma, L. H. Tan, X. J. Zhao, Y. S. Wang, H. M. Ma, and D. Zhang, "Relationship between the change of temperature and the contents of CGRP and at II in the tissues along the meridian in rabbits," Acupuncture Research, no. 2, pp. 149-151, 2002.

[36] D. Y. Cao, H. Z. Niu, and Y. Zhao, "Stimulation of acupoint induces release of substance P through primary afferent reflex," Chinese Acupuncture \& Moxibustion, vol. 21, no. 10, pp. 623-625, 2001.

[37] S.-X. Ma, "Enhanced nitric oxide concentrations and expression of nitric oxide synthase in acupuncture points/meridians," Journal of Alternative and Complementary Medicine, vol. 9, no. 2, pp. 207-215, 2003.

[38] T.-T. Chang, K.-C. Chen, K.-W. Chang et al., "In silico pharmacology suggests ginger extracts may reduce stroke risks," Molecular BioSystems, vol. 7, no. 9, pp. 2702-2710, 2011.
[39] C. Y. C. Chen, "A novel integrated framework and improved methodology of computer-aided drug design," Current Topics in Medicinal Chemistry, vol. 13, no. 9, 2013.

[40] C. Y.-C. Chen, "Computational screening and design of traditional Chinese medicine (TCM) to block phosphodiesterase-5," Journal of Molecular Graphics and Modelling, vol. 28, no. 3, pp. 261-269, 2009.

[41] X. M. Li, Y. Q. Li, J. Z. Chen et al., "The influence of skin microcirculation blood perfusion at Zusanli acupoint by stimulating with lift-thrust reinforcing and reducing acupuncture manipulation methods on healthy adults," Evidence-Based Complementary and Alternative Medicine, vol. 2013, Article ID 452697, 7 pages, 2013.

[42] G. J. Wang, J. G. Han, G. Litscher, and W. B. Zhang, "System identification algorithm analysis of acupuncture effect on mean blood flux of contralateral Hegu acupoint," Evidence-Based Complementary and Alternative Medicine, vol. 2012, Article ID 951928, 7 pages, 2012.

[43] Z. Zheng, Y. Liu, Y. Guo et al., "Preliminary exploration of research method for studying the influence of acupuncture manipulations on electrical signals of spinal dorsal root nerve in rats," in Proceedings of the 6th International Conference on Natural Computation (ICNC'10), pp. 509-513, August 2010. 


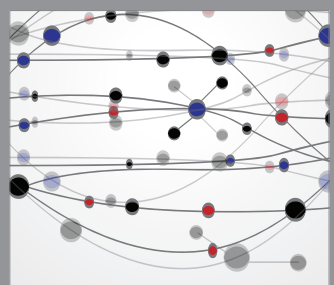

The Scientific World Journal
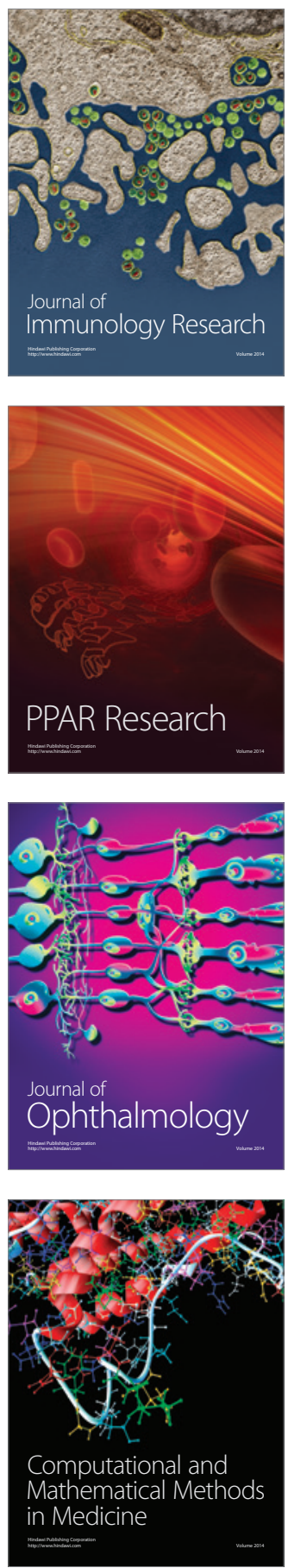

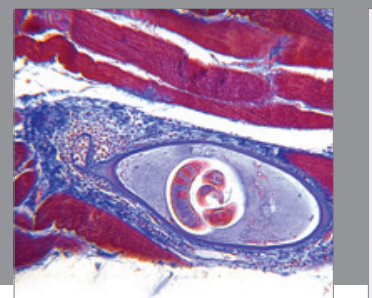

Gastroenterology

Research and Practice
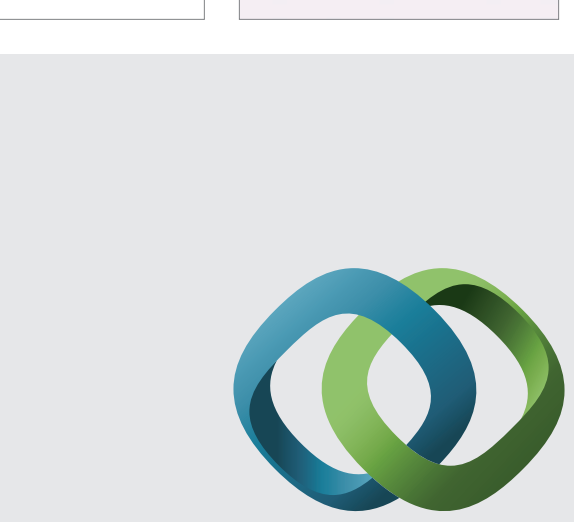

\section{Hindawi}

Submit your manuscripts at

http://www.hindawi.com
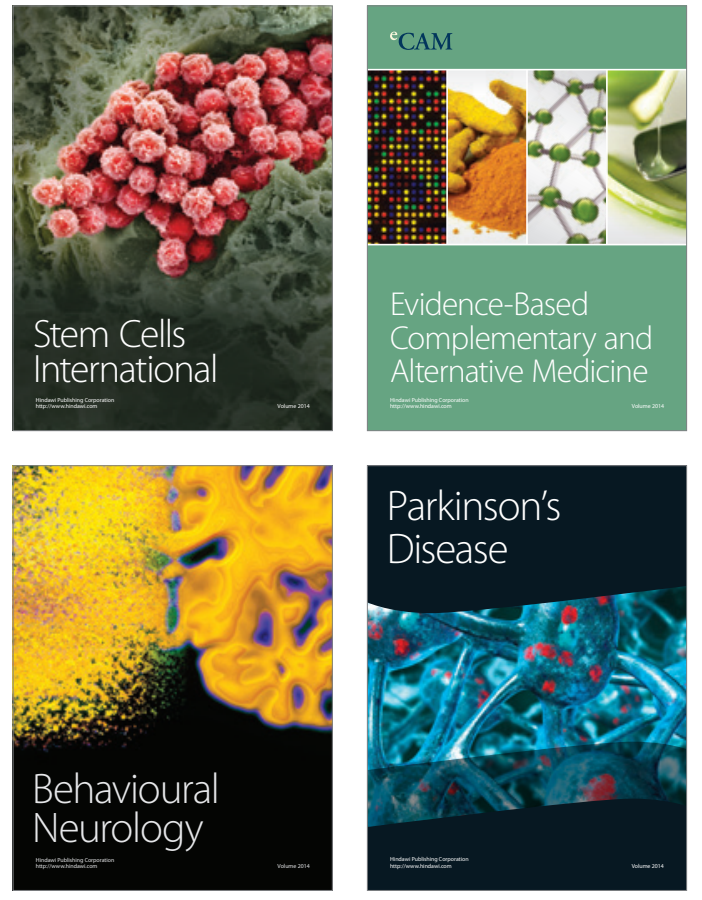
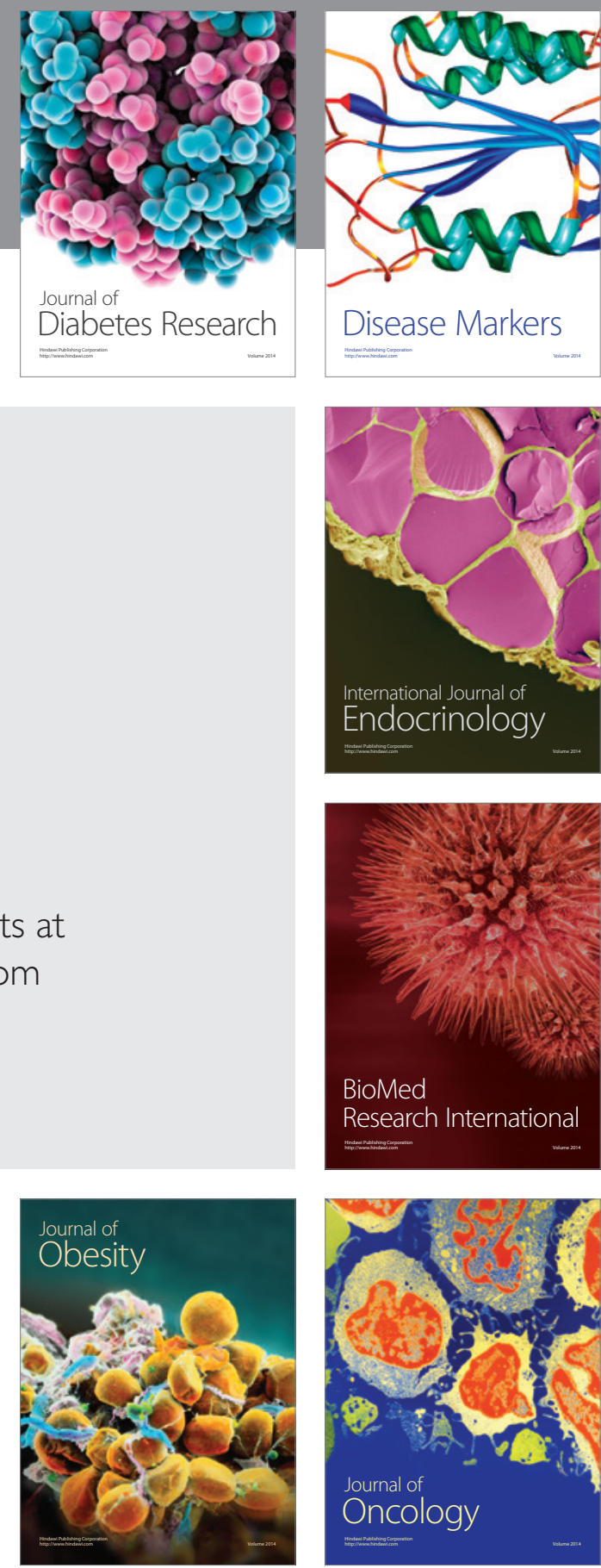

Disease Markers
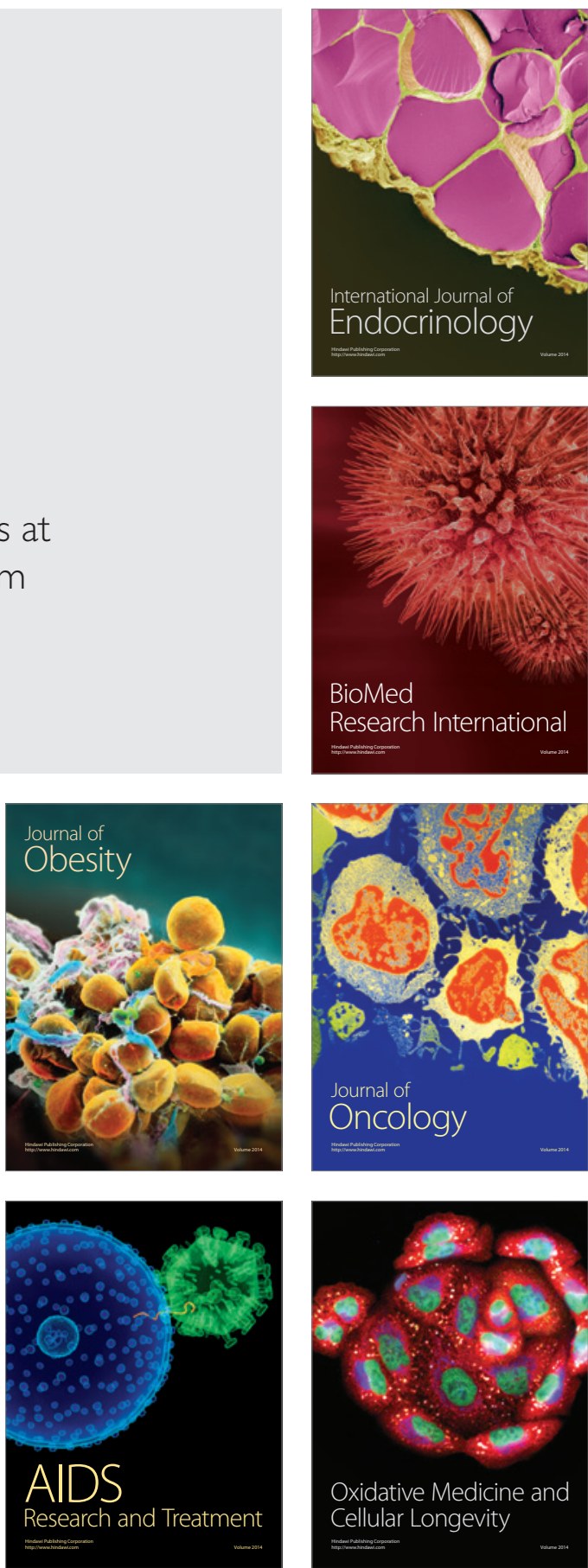